\title{
ON THE TOTAL ELECTRONIC DENSITY OF STATES OF DISORDERED SOLIDS
}

\author{
M. A. GRADO-CAFFARO and M. GRADO-CAFFARO
}

C/Julio Palacios 11, 9으 28029-Madrid, Spain

(Received 5 April 1997; In final form 22 July 1997)

\begin{abstract}
The concept of total electronic density of states calculated for small clusters in amorphous solids is presented in a new way by using a mathematical formulation based upon matrix calculus. Furthermore, the associated Green's function is discussed in a special form. Our results can be used to study amorphous solids as a-Ge and a-Si.
\end{abstract}

Keywords: Total electronic density of states; small clusters; disordered solids

\section{INTRODUCTION}

It is well-known that amorphous tetrahedrally bonded semiconductors play an important role in the context of disordered solids. These semiconductors exhibit electric, optical and magnetic properties that to date are not completely understood in spite of the large amount of work developed by using experimental techniques and somewhat obscure theoretical methods. Consequently, research efforts to develop powerful theoretical methods are necessary. In particular, electronic density of states as well as phonon density of states are tools to evaluate some of the above properties that become indispensable to perform calculations. In the following, we will reexamine total electronic density of states from a special point of view; the associated Green's function will be also considered. 


\section{THEORETICAL CONSIDERATIONS}

First, we start from the well-known expression for the local density of states:

$$
g(\vec{r}, E)=\sum_{n}\left[\left|\psi_{n}(\vec{r})\right|^{2} \delta\left(E-E_{n}\right)\right]
$$

where $\psi_{n}$ and $E_{n}$ are eigenfunctions and eigenvalues, respectively, corresponding to the time-independent Schrödinger equation, namely:

$$
\hat{H}\left|\psi_{n}\right\rangle=E_{n}\left|\psi_{n}\right\rangle
$$

On the other hand, notice that $\vec{r}$ denotes position vector and $\delta$ Dirac delta function. Now, we formulate the total electronic density of states for an amorphous cluster, namely:

$$
\tilde{g}(E)=\int_{D} g(\vec{r}, E) d^{3} \vec{r}
$$

In Refs. [1], [2] approximate formulae for $\tilde{g}(E)$ have been given by employing Taylor series. However, for very small clusters, the following relationship is valid (see Refs. [3], [4]):

$$
\tilde{g}(E) \approx \Omega g(\vec{o}, E)
$$

where $\Omega$ is the volume of the considered cluster.

It is feasible to consider Eq. (4) to treat problems related to 5-atom clusters of a-Ge and a-Si (see Refs. [3-6]). At this point, we wish to concentrate on this equation in order to obtain a new formulation; to get this end, we have [3]:

$$
g_{i j}(\vec{r}, E)=\sum_{n}^{5}\left[\left|\int_{R^{\prime}} \psi_{n}\left(\vec{r}^{\prime}\right) \phi_{i j}\left(\vec{r}^{\prime}-\vec{r}\right) d^{3} \vec{r}^{\prime}\right|^{2} \delta\left(E-E_{n}\right)\right]
$$


where $\phi_{i j}$ are molecular bonding orbitals referring to the atoms labelled $i$ and $j$ (see Ref. [3]). Then, by using formula (4), we get:

$$
\tilde{g}_{i j}(E) \approx \Omega \sum_{n}^{5}\left[\left|\int_{R^{\prime}} \psi_{n}\left(\vec{r}^{\prime}\right) \phi_{i j}\left(\vec{r}^{\prime}\right) d^{3} \vec{r}^{\prime}\right|^{2} \delta\left(E-E_{n}\right)\right]
$$

when $\tilde{g}_{i j}$ becomes a matrix element; thus, the relation between $\tilde{g}_{i j}$ and $\phi_{i j}$ is clear. Therefore, we can speak in terms of the matrices $\tilde{\boldsymbol{G}}$ and $\boldsymbol{\Phi}$ with

$$
\tilde{\boldsymbol{G}}=\left(\tilde{g}_{i j}\right) ; \quad \boldsymbol{\Phi}=\left(\phi_{i j}\right) ; \quad i, j=1,2, \ldots, 5
$$

On the other hand, the above definite integral can be expressed as a scalar product $\langle\mid\rangle$ so that we have:

$$
\tilde{g}_{i j}(E) \approx \Omega \sum_{n}^{5}\left[\left|\left\langle\psi_{n}^{*} \mid \phi_{i j}\right\rangle\right|^{2} \delta\left(E-E_{n}\right)\right]
$$

Finally, we shall briefly consider the Green's function associated with the local electronic density of states; this local Green's function satisfies the following relationship, namely (see, for example, Ref. [3]):

$$
g(\vec{r}, E)=-\frac{1}{\pi} \operatorname{Im} \mathcal{G}(\vec{r}, E)
$$

so that:

$$
\begin{aligned}
\operatorname{Im} \mathcal{G}_{i j}(\vec{r}, E) & =-\pi g_{i j}(\vec{r}, E) \\
& =-\pi \sum_{n}\left[\left|\int_{R^{\prime}} \psi_{n}\left(\vec{r}^{\prime}\right) \phi_{i j}\left(\vec{r}^{\prime}-\vec{r}\right) d^{3} \vec{r}^{\prime}\right|^{2} \delta\left(E-E_{n}\right)\right]
\end{aligned}
$$

Then, by taking into account Eq. (9), it is inferred:

$$
\mathcal{G}_{i j}^{*}(\vec{o}, E)-\mathcal{G}_{i j}(\vec{o}, E)=2 \pi i \sum_{n}\left[\left|\left\langle\psi_{n}^{*} \mid \phi_{i j}\right\rangle\right|^{2} \delta\left(E-E_{n}\right)\right]
$$

It is clear that local Green's function adopts a matrix character. 


\section{CONCLUDING REMARKS}

Previous formulation is useful to study very small clusters of a-Ge and a-Si by computer programs employing some advanced scientific languages. On the other hand, we can claim that, although large clusters become difficult from the theoretical point of view, a quasi exact picture for small clusters is very useful to understand some essential features of large clusters. Moreover, we want to emphasize that advanced analytical methods are urgent to consider the above problems.

\section{References}

[1] Grado-Caffaro, M. A. and Grado-Caffaro, M. (1993). Active and Pass. Electron. Comp., 16, 49-53.

[2] Grado-Caffaro, M. A. and Grado-Caffaro, M. Act. Pass. Electron. Comp. (in press).

[3] Grado-Caffaro, M. A. and Grado-Caffaro, M. (1994). Act. Pass. Electron. Comp., 16, $105-107$

[4] Tong, B. Y. (1974). AIP Conf. Proc., 20, 148.

[5] Choo, F. C. (1973). Bull. Am. Phys. Soc., 18, 1588.

[6] Henderson, D. (1973). Phys. Status Solidi (b), 57, 661. 

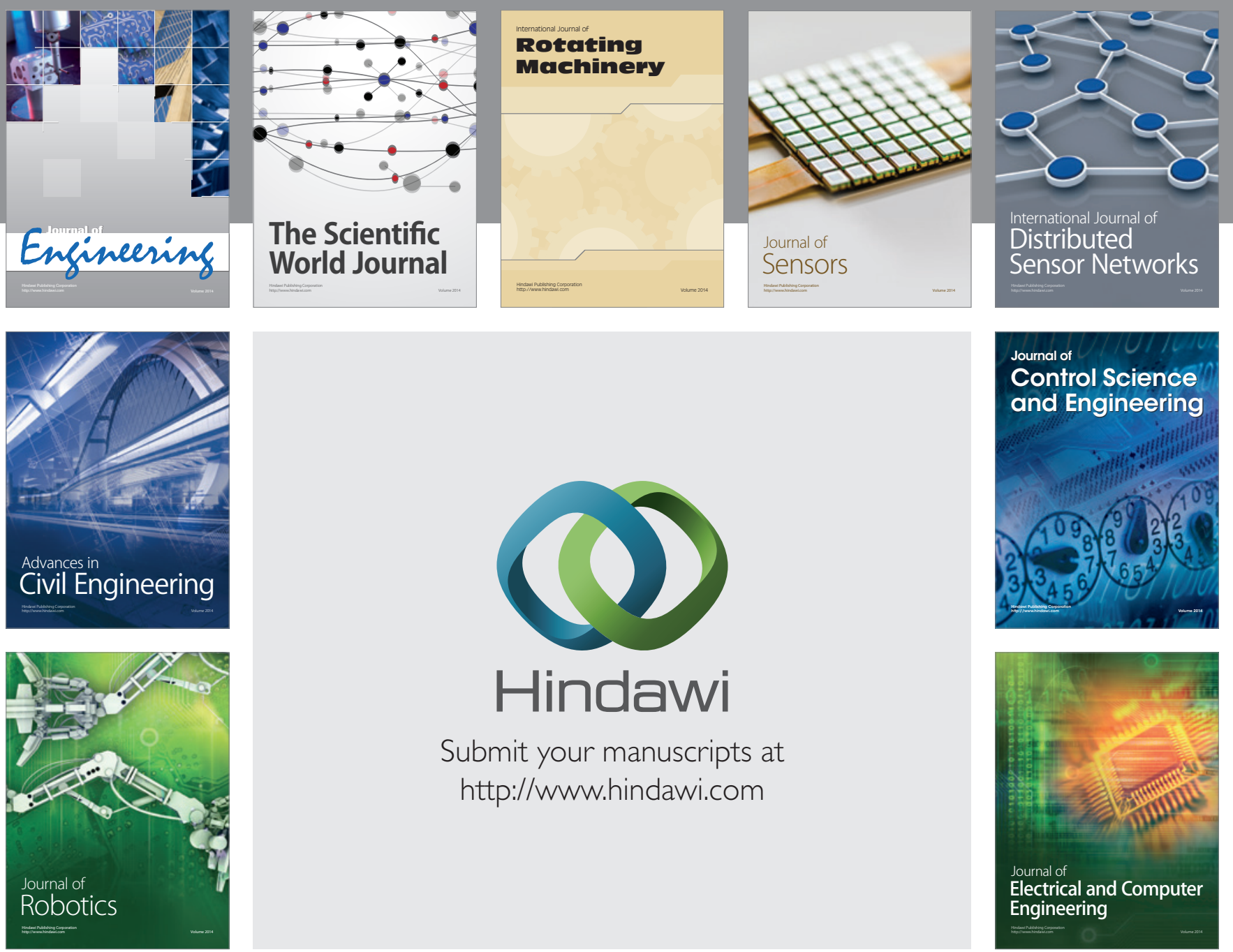

Submit your manuscripts at

http://www.hindawi.com
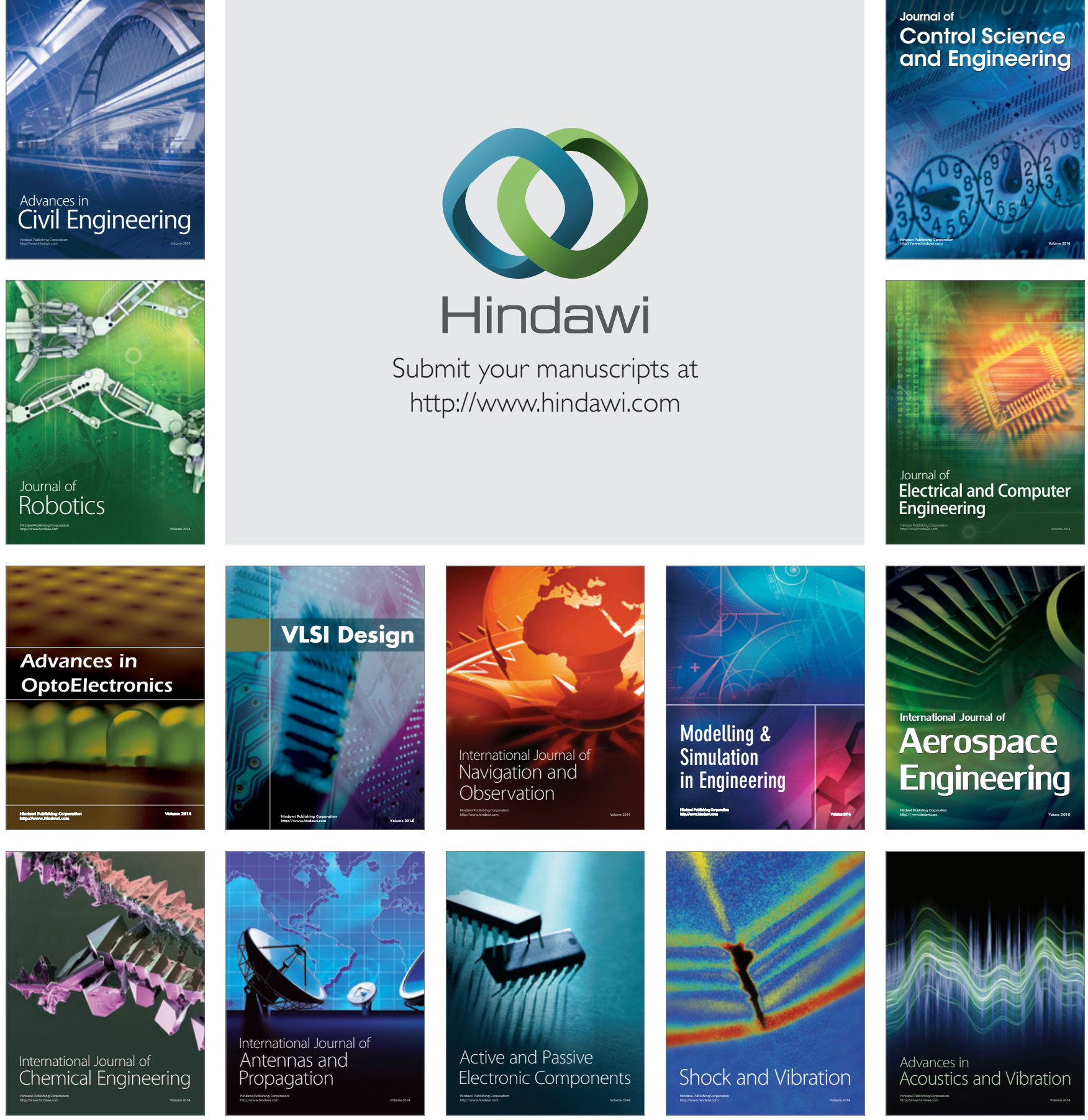